University of South Carolina

Scholar Commons

$5-15-1988$

\title{
Experimental study of nonlinear conductance in small metallic samples
}

Richard A. Webb

University of South Carolina - Columbia, webbra@mailbox.sc.edu

S. Washburn

C. P. Umbach

Follow this and additional works at: https://scholarcommons.sc.edu/phys_facpub

Part of the Physics Commons

\section{Publication Info}

Published in Physical Review B, Volume 37, Issue 14, 1988, pages 8455-8458.

Webb, R.A., Washburn, S., and Umbach, C.P. (1988). Experimental study of nonlinear conductance in small metallic samples. Physical Review B, 37(14), 8455-8458. doi: 10.1103/PhysRevB.37.8455

(C) 1988 The American Physical Society.

This Article is brought to you by the Physics and Astronomy, Department of at Scholar Commons. It has been accepted for inclusion in Faculty Publications by an authorized administrator of Scholar Commons. For more information, please contact digres@mailbox.sc.edu. 


\title{
Experimental study of nonlinear conductance in small metallic samples
}

\author{
R. A. Webb, S. Washburn, and C. P. Umbach \\ IBM Thomas J. Watson Research Center, P.O. Box 218, Yorktown Heights, New York 10598
}

(Received 12 January 1988)

\begin{abstract}
We have directly observed current-dependent, nonlinear contributions to the conductance fluctuations of phase-coherent metallic wires and loops. The fluctuations in the current-voltage curves are reproducible, asymmetric about $I=0$, and in qualitative agreement with theoretical predictions. In ac measurements, the nonlinear terms also generate large harmonic signals of the conductance fluctuations whose dependence on the drive current can be understood qualitatively. The spectra of harmonics from loops and wires have different dependences on the voltage across the sample.
\end{abstract}

In the past two years, the study of quantuminterference effects in systems where the electron phasecoherence length is comparable to the size of the sample has revealed several new and interesting results. The prediction that the Aharonov-Bohm effect can be observed in transport measurements on samples where the carriers propagate diffusively ${ }^{1}$ has been confirmed. ${ }^{2}$ The experimental observations of aperiodic fluctuations ${ }^{3}$ in wires and $h / e$ periodic Aharonov-Bohm oscillations ${ }^{4}$ in loops have forced an additional theoretical effort ${ }^{5-7}$ to understand conductance in diffusive systems where the phase coherence of the carriers is retained over distances $L_{\phi}$ which are as large or larger than the sample length $L$. This in turn has led to the "universal" conductancefluctuation theory ${ }^{6,7}$ where it has been shown that the rms value of the conductance fluctuations, obtained as either the Fermi energy or the magnetic field is varied, is $\langle\Delta G\rangle \sim e^{2} / h$ in any phase-coherent device $\left(L_{\phi} \sim L\right)$. This was in agreement with existing data ${ }^{3}$ and has been experimentally confirmed in various ways since..$^{8-11}$

When the electrons retain their phase coherence, the measured interference properties are extremely sensitive to all the details of the impurity potential. If the average voltage developed across a section of the sample $L_{\phi}$ long is changed, the interference properties are also affected. This leads to voltage-dependent fluctuations in the conductance that are similar to effects seen when the vector potential is changed and are quite different from normal electron heating effects. In this paper we present experimental data for wires and rings which clearly demonstrate that small structures exhibit a wealth of new and unexpected nonlinear effects.

Al'tshuler and Khmel'nitskii ${ }^{12}$ and Larkin and Khmel'nitskii ${ }^{13}$ have developed a theory for small samples in the diffusive regime which predicts that the current-voltage $(I-V)$ curve is not linear due to quantum interference effects. That is, the dimensionless conductance $g(V) \equiv G(V) /\left(e^{2} / h\right)$ is not constant, but rather, a random function of $V$ described by the correlation function $K_{g}(g(V) g(V+\Delta V))$. They predict that the characteristic voltage scale on which $g$ fluctuates is $V_{C}=\hbar D / e L^{2}$. When $V \ll V_{C}$, the fluctuations in the $I-V$ curves are small, but as $V$ approaches $V_{C}$ the fluctuations are $\Delta g=g-\langle g\rangle \sim 1$. When the voltage across the sample exceeds $V_{C}$, the fluctuations decay on average as $\left(V_{C} / V\right)^{1 / 2}$. In addition they predict that, because these systems lack inversion-center symmetry, the $I-V$ curves will be asymmetric about zero voltage with $[g(V)-g(-V)]^{2} \sim 1$. (Some of these effects have been observed in experiments on $\mathrm{Si}$ metal oxide semiconductor field effect transistors. ${ }^{14}$ ) In ac measurements, as we will show, this asymmetry in the $I-V$ curves leads to the generation of unexpectedly large signals at all the harmonics. $^{12}$

The experiments reported here involve three samples - a polycrystalline Au loop $(0.03 \mu \mathrm{m}$ thick, 0.6 $\mu \mathrm{m}$ inside diameter, and $0.09 \mu \mathrm{m}$ linewidth) with six probes equally spaced around the perimeter, a $0.08 \mu \mathrm{m}$ thick, $0.1 \mu \mathrm{m}$ wide Sb wire with eight voltage probes, and a $0.03 \mu \mathrm{m}$ wide $\mathrm{Au}$ wire with six voltage probes. All samples were made from high-purity (0.999 999) starting material using electron-beam lithography and negative resist. The four-wire conductance of the samples was measured by phase-sensitive detectors at frequencies in the range $40<\omega<160 \mathrm{~Hz}$. The differential conductance was measured by applying both a small constant $10-\mathrm{nA}$ rms current at frequency $\omega$ and a dc current to the sample and detecting the voltage at $\omega$ as a function of the dc current. The harmonic content of the fluctuations was measured in the absence of dc current by driving the current through the device at the fundamental frequency $\omega$ and detecting at the harmonics $N \omega(N=2,3, \ldots)$.

In Fig. 1, we display the results of a direct differential conductance measurement (at $T=0.01 \mathrm{~K}$ and $H=3.0 \mathrm{~T}$ ) as a function of the dc current in the $\mathrm{Sb}$ wire using two voltage probes separated by $0.6 \mu \mathrm{m}$. Similar results were obtained at other values of the magnetic field and on $\mathrm{Au}$ wires. The differential conductance $G=\partial I / \partial V$ is constant in Ohmic samples. The data in Fig. 1(a), however, clearly show that the conductance is a randomly fluctuating quantity. The average conductance $(\langle G\rangle$ $=1 / 48.5 \Omega$ ) is independent of the dc current and has been subtracted from the data. The peak-to-peak fluctuations are as large as $\Delta g=4$ when $|I|<1 \mu \mathrm{A}$, and they decrease in magnitude as the current increases. The characteristic current scale of the fluctuations increases as $|I|$ increases. In addition, $\Delta g=g(I)-\langle g\rangle$ is not symmetric about zero current, $\Delta g(I) \neq \Delta g(-I)$.

The inset displays the autocorrelation function $C(\Delta I)$ of the data in Fig. 1(a). For these data $C(0)=\left\langle(\Delta g)^{2}\right\rangle \simeq 0.23$. $C(\Delta I)$ decays rapidly near $\Delta I=0$ with a characteristic current scale of $30 \mathrm{nA}$ 


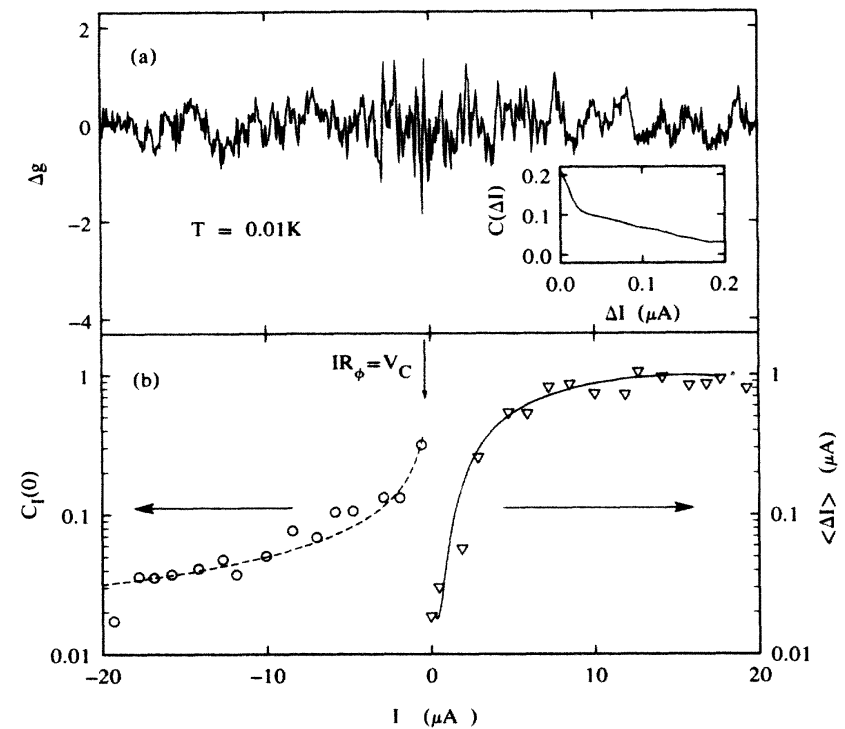

FIG. 1. (a) The fluctuations in the differential conductance $\Delta g$ of an $\mathrm{Sb}$ wire as a function of the dc current through the wire at $T=0.01 \mathrm{~K}$. Inset, the auto correlation $C(\Delta I)$. (b) The autocorrelation function $C_{I}(0)(O)$ and current correlation scale $(\nabla)$ of the data in (a) for narrow regions centered around the current marked by each point. The dashed line is Eq. (2) with $T=0.01 \mathrm{~K}$, and the solid line guides the eye. The arrow marks $I=V_{C} / R_{\phi}$.

$\sim k_{B} T / e R_{\phi}\left(R_{\phi}\right.$ is the resistance of a segment of length $L_{\phi}-98 \Omega$ for this wire), but as $\Delta I$ increases beyond 30 $\mathrm{nA}, C(\Delta I)$ decays more slowly. Very similar correlation scales have been measured in Au wires where $V_{C}$ is an order of magnitude smaller, but $R_{\phi}$ is again $100 \Omega$. Calculations by Hershfield ${ }^{15}$ have shown that, at $T=0, K_{g}$ decays on a voltage scale of $0.03 V_{C}$ or approximately $10 \mathrm{nA}$ for this sample, which agrees reasonably well with correlation scales in $\mathrm{Sb}\left(30 \mathrm{nA} \simeq 0.1 V_{C} / R_{\phi}\right)$, but not so well with the data from Au wires. Since $C(\Delta I)$ averages over the entire curve, it is not useful for extracting the current dependence of the fluctuations. Under the conditions $L_{\phi}=L$ and $k_{B} T \ll e V_{C} \ll \Delta V, K_{g}$ takes the form ${ }^{13}$

$K_{g}(V, \Delta V)=\frac{-V}{\pi^{2} V_{c}}\left[\operatorname{coth}\left[\frac{e V}{k_{B} T}\right]-\frac{k_{B} T}{e V}\right] F\left(\frac{\Delta V}{V_{C}}\right)$,

where $V$ is the voltage at the center of the line segment, $\Delta V=I R$ is the voltage difference across the line segment, and $F\left(\Delta V / V_{C}\right)$ is a complicated integral-differential equation. We approximate ${ }^{13} F\left(\Delta V / V_{C}\right) \simeq\left(V_{C} / I R\right)^{5 / 3}$ so that $K_{g}$ can be rewritten as

$K_{g}(I)=A\left[\operatorname{coth}\left[\frac{e I R}{2 k_{B} T}\right]-\frac{2 k_{B} T}{e I R}\right]\left[\frac{I R}{V_{C}}\right]^{-2 / 3}$,

where $R$ is the average resistance of the line segment under consideration, and $A$ is a numerical constant on the order of $1 / \pi^{2}$. This function is zero near zero current ( $G=$ const) and reaches a maximum when $e I R=2 k_{B} T$ and then decays as $\left(I R / V_{C}\right)^{-2 / 3}$. At higher voltages, the fluctuations in the current-voltage curves decay because $\Delta V / V_{C}$-independent fluctuation patterns contribute to
$\Delta g$. For voltage probe spacing $L<L_{\phi}$, the relevant sample length is $L_{\phi},{ }^{11,16-18}$ and $R$ in Eq. (2) should be the resistance $R_{\phi}$ across a phase-coherent segment of the sample. The theory which led to Eq. (1) ignored any heating of the carriers by the applied voltage. When electron heating effects are included in the theory, ${ }^{13}$ the correlation scale of the fluctuations increases, and the magnitude of $K_{g}(I)$ decreases more rapidly than Eq. (2).

In order to compare our data with $K_{g}$, we divide the current scale of the data displayed in Fig. 1(a) into small, overlapping intervals and compute the autocorrelation function $C_{I}(\Delta I)$ for each current interval centered at $I$. In order to obtain a reasonable "ensemble average" from one trace $\Delta g(I)$, the analysis is repeated for various interval widths centered at various currents, and all of the resulting $C_{I}(0)$ are averaged together. The current dependence of the maximum amplitude $C_{I}(0)$ [circles in Fig. 1(b)] agrees qualitatively with the theoretical prediction (dashed line) for $K_{g}$ from Eq. (2). [The scatter in the data is representative of the statistical errors in the averaged $C_{I}(0)$.] The width of the $C_{I}(0)$ (triangles) grows rapidly for $I>500 \mathrm{nA}$ (being constant for smaller currents) to a value which is approximately 50 times larger than near $I=0$ and depends weakly on current for $5 \lesssim I<20 \mu \mathrm{A}$. In contrast, over the entire range of current, $C_{I}(0)$ decays smoothly as $I^{-2 / 3}$. According to the theory, ${ }^{13}$ the increase of the fluctuation scale is the signature that $L_{\phi}$ decreases as the current increases. We have estimated the temperature difference between the sample and the surrounding liquid helium by including electron thermal conduction, heat links into the $\mathrm{Si}_{3} \mathrm{~N}_{4} / \mathrm{Si}$ substrate, and the Kapitza boundary resistances. We find that the temperature of the electrons is probably above $\sim 1 \mathrm{~K}$ at dc currents $I>5 \mu \mathrm{A}$. From weak localization and from studies of the length dependence of the nonlocal conductance fluctuations, ${ }^{19}$ the value of $L_{\phi}$ was determined to be $L_{\phi} \simeq 1.2 \pm 0.2 \mu \mathrm{m}$ for $T<0.2 \mathrm{~K}$ and $L_{\phi} \propto T^{-3 / 4}$ at higher temperature so that $L_{\phi} \simeq 0.5 \mu \mathrm{m}$ at $2 \mathrm{~K}$. If the increase in the correlation scale were purely the measure of $V_{C}=\hbar D / e L_{\phi}^{2}$ increasing, then we would infer that at our highest currents $L_{\phi}<0.2 \mu \mathrm{m}$ or that the sample temperature is $T>7 \mathrm{~K}$. The respectable agreement between the $C_{I}(0)$ and Eq. (2), which presumes no heating, indicates that Eq. (2) is rather weakly dependent on the carrier temperature once the dependence of $V_{C}$ on $L_{\phi}$ is included.

An alternative method of studying the nonlinear components of the conductance is to make use of the "rectifying" nature of the potential disorder and measure the harmonics generated by the sample. ${ }^{12}$ For an ac measurement of the conductance of a linear element at frequency $\omega$, the signal at $2 \omega$, etc., is zero. If instead the sample is nonlinear, then there will be power generated at the harmonics of $\omega$. (This is distinct from the signature of heating which appears only at odd harmonics in samples where $R$ depends on $T$.) A series of such harmonic measurements was made for the same $\mathrm{Sb}$ wire discussed above. The experimental procedure was to measure the voltage difference developed across one segment of the sample at the fundamental and two harmonics $(\omega, 2 \omega$, 
and $4 \omega$, for example) simultaneously as a function of magnetic field. Examples of the Fourier transforms of the voltage fluctuations observed at frequency $\omega$ (solid curve) and at frequency $2 \omega$ (dashed curve) are displayed in Fig. 2(a) for the same $0.6-\mu \mathrm{m}$-long line segment discussed above. The random voltage fluctuations $\Delta V$ are centered around $485 \mathrm{nV}(I=10 \mathrm{nA})$ for the fundamental and $0 \mathrm{nV}$ for the second harmonic. Cross correlation of the two traces shows that the voltage fluctuations at $\omega$ and $2 \omega$ are uncorrelated. The rms value of the second harmonic signal is only a factor of $\sim 4$ smaller than that at the fundamental.

In Fig. 2(b) we show the results of a detailed study of the current dependence of the harmonic content, measured at $\omega, 2 \omega, 3 \omega, 4 \omega$, and $6 \omega$, of the voltage fluctuations. At low drive currents, $I<k_{B} T / e R_{\phi}$ the rms signal at the fundamental or any harmonic is proportional to the current. At higher current, the amplitude grows as $I^{1 / 2}$, as expected from energy averaging over uncorrelated voltage fluctuation patterns. We believe that there are no severe electron heating effects contained within these data because the average value of the fundamental fluctuations would grow more rapidly than $I^{1 / 2}$ if $L_{\phi}$ were decreasing. For the fundamental, the value of the current where the power law changes from $I$ to $I^{1 / 2}$ is $I \sim k_{B} T / e R_{\phi}$. The current at which the power law changes is larger for each successive harmonic. This increase of the crossover with harmonic number $N$ is unexpected and not understood at present. The low current behavior $[\langle\Delta V(N \omega)\rangle \propto I]$ of the harmonic content of the fluctuations is unexpected in the following sense. For a diode, or some other grossly nonlinear element, $\langle V(N \omega)\rangle \propto I^{N}$. We, however, are measuring the harmonic content of the conductance fluctuations. The proportionality $\langle\Delta V(N \omega)\rangle \propto I$ can be understood with the aid of a simple model for fluctuating resistance. ${ }^{20}$ It is possible that, at small enough bias current, the average voltage at $2 \omega$ for fixed magnetic field does increase as $I^{2}$ but for our samples this signal is much too small to measure.

Figure 2(c) demonstrates another remarkable feature of $\langle\Delta V(N \omega)\rangle$. We display the rms value $\langle\Delta V\rangle$ obtained at two fixed currents as a function of $N$ for $1 \leq N \leq 10$. At high currents $\langle\Delta V\rangle$ decays as $N^{-1}$. If this were to continue for all the higher harmonics, the total $\langle\Delta V\rangle$ defined as the average of the sum of $\Delta V$ at all harmonics, $\sum_{N=1}^{\infty} \Delta V(N \omega)$, would diverge. Since the fluctuations at different harmonics are uncorrelated, the total can be estimated from

$$
\langle\Delta V\rangle=\left(\sum_{N=1}^{\infty}[\Delta V(N \omega)]^{2}\right]^{1 / 2}=1.28 V(\omega) .
$$

Therefore $\langle\Delta g\rangle$ measured at dc should be $30 \%$ larger than obtained in an ac measurement at a single frequency. At excitations $I<k_{B} T / e R_{\phi}$ the decay of $\langle\Delta V\rangle$ with increasing $N$ is much stronger. In this range, less of a discrepancy between a dc measurement and an ac measurement would be expected.

Similar harmonic measurements were also performed on a loop of gold (shown in the inset of Fig. 3). This sample allows the study of the nonlinear effects in a different
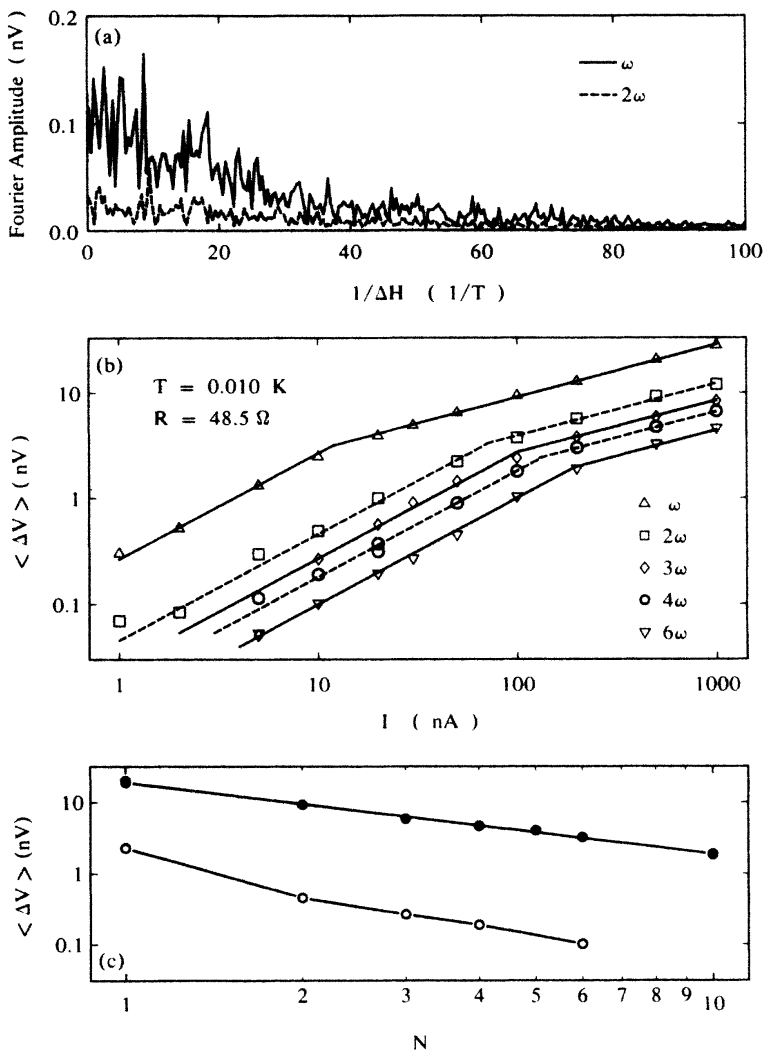

FIG. 2. (a) The Fourier transform of voltage fluctuations measured at the driving frequency $\omega$ (solid curve) and at its first harmonic (dashed curve) in a wire. The drive current is $10 \mathrm{nA}$. (b) $\langle\Delta V(N \omega)\rangle$ as a function of drive current at $T=0.01 \mathrm{~K}$. (c) $\langle\Delta V(N \omega)\rangle$ as a function of harmonic number $N$ for $I=500 \mathrm{nA}$ (๑) and for $I=10 \mathrm{nA}(O)$.

material and for $h / e$ Aharonov-Bohm oscillations as well. A synopsis of the measured $\langle\Delta V\rangle$ appears in Fig. 3. The aperiodic fluctuations behave just as in the $\mathbf{S b}$ wire: the amplitude is proportional to $I^{1 / 2}$ at high current and proportional to $I$ at low current. The point at which the power law changes from $I$ to $I^{1 / 2}$ is shifted toward higher current for the $2 \omega$. At high currents, $\langle\Delta V(2 \omega)\rangle$ is about a factor of 4 smaller than $\langle\Delta V(\omega)\rangle$ (which contrasts with the factor of 2 in the Sb wire). For the periodic oscillations there is a different result. For the fundamental frequency, the fluctuation amplitude is proportional to $I^{1 / 2}$ down to the lowest currents studied. The absence of the linear part is somewhat perplexing because in all our previous experiments, the $h / e$ voltage oscillations were found to increase linearly with increasing current at low currents.

For this geometry we expect that $V_{C}$ will be approximately the same for the $h / e$ oscillations as for the aperiodic fluctuations: $V_{C} \sim 2 \mu \mathrm{V}$ [approximately 300 $n A$ for the data in Fig. 3(a)]. (Also, $e V_{C} \sim k_{B} T$ for these data, which were obtained at $T=0.04 \mathrm{~K}$.) For this reason the difference in break point between the aperiodic fluctuation data and the $h / e$ data is a puzzle. If we assume that the same relative positions of the break points for the aperiodic fluctuations will repeat for the $h / e$ oscillations, we estimate that the break in the power law for 


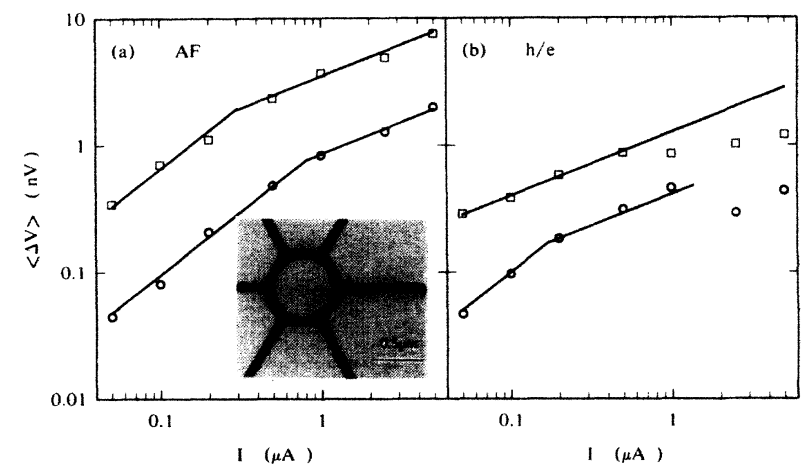

FIG. 3. (a) $\langle\Delta V\rangle$ as a function of the drive current $I$ at the fundamental $(\square)$ and first harmonic $(O)$ for the aperiodic fluctuations. Inset is the measured $0.58 \mu \mathrm{m}$ inside diameter gold loop with six equally spaced probes. (b) $\langle\Delta V\rangle$ as a function of $I$ at the fundamental $(\square)$ and first harmonic $(O)$ for the $h / e$ oscillations.

the fundamental $h / e$ data would occur near or below the lowest current studied. We also see that at currents greater than $1 \mu \mathrm{A}$ the fundamental $h / e$ voltage signal is nearly independent of current and the signal at the second harmonic decreases slightly with increasing current. It is likely that this change is not associated with a decrease in $L_{\phi}$ because the aperiodic fluctuations continue to follow the $I^{1 / 2}$ dependence. It appears to indicate a real difference between the current dependence of the $h / e$ oscillations and that of the aperiodic fluctuations.

In summary, we find that, in qualitative agreement with recent theories, the $I-V$ curves of small $\mathrm{Sb}$ and $\mathrm{Au}$ wires are asymmetric about zero current and nonlinear down to very small voltages. In an ac measurement, these nonlinearities allow for the generation of conductance fluctuations at higher harmonics of the drive frequency which decay slowly with increasing harmonic number. The harmonic signals generated by wires are qualitatively different from those generated by rings.

We acknowledge useful conversations with B. Al'tshuler, D. Divincenzo, S. Hershfield, D. Khmel'nitskii, and R. Landauer.
${ }^{1}$ B. L. Al'tshuler, A. G. Aronov, and B. Z. Spivak, Pis'ma Zh. Eksp. Teor. Fiz. 33, 101 (1981) [JETP Lett. 33, 94 (1981)]; Y. Gefen, Y. Imry, and M. Ya. Azbel, Phys. Rev. Lett. 52, 129 (1984); M. Büttiker, Y. Imry, R. Landauer, and S. Pinhas, Phys. Rev. B 31, 6207 (1985).

${ }^{2}$ D. Yu. Sharvin and Yu. V. Sharvin, Pis'ma Zh. Eksp. Teor. Fiz. 34, 285 (1981) [JETP Lett. 34, 272 (1981)]; A. G. Aronov and Yu. V. Sharvin, Rev. Mod. Phys. 59, 755 (1987), and references therein.

${ }^{3}$ R. A. Webb, S. Washburn, C. P. Umbach, and R. B. Laibowitz, in Localization, Interaction, and Transport Phenomena in Impure Metals, edited by G. Bergmann, Y. Bruynseraede, and B. Kramer (Springer, Heidelberg, 1985); C. P. Umbach, S. Washburn, R. B. Laibowitz, and R. A. Webb, Phys. Rev. B 30, 4048 (1984).

${ }^{4}$ R. A. Webb, S. Washburn, C. P. Umbach, and R. B. Laibowitz, Phys. Rev. Lett. 54, 2696 (1985); V. Chandrasekhar, M. J. Rooks, S. Wind, and D. E. Prober, ibid. 55, 1610 (1985); S. Datta et al., ibid. 55, 2344 (1985).

${ }^{5}$ A. D. Stone, Phys. Rev. Lett. 54, 2692 (1985).

${ }^{6}$ B. L. Al'tshuler, Pis'ma Zh. Eksp. Teor. Fiz. 41, 530 (1985) [JETP Lett. 41, 648 (1985)]; B. L. Al'tshuler, V. E. Kravtsov, and I. V. Lerner, Zh. Eksp. Teor. Fiz. 91, 2276 (1986) [Sov. Phys. - JETP 64, 1352 (1986)], and references cited therein.

${ }^{7}$ P. A. Lee, A. D. Stone, and H. Fukuyama, Phys. Rev. B 35, 1039 (1987).

${ }^{8}$ S. Washburn and R. A. Webb, Adv. Phys. 35, 375 (1986), and references cited therein.

${ }^{9}$ A. D. Benoit, S. Washburn, C. P. Umbach, R. B. Laibowitz, and R. A. Webb, Phys. Rev. Lett. 57, 1765 (1986).

${ }^{10}$ M. Büttiker, Phys. Rev. Lett. 57, 1761 (1986).

${ }^{11}$ Y. Isawa, H. Ebisawa, and S. Maekawa, J. Phys. Soc. Jpn. 56, 25 (1987); Proceedings of the Conference on Anderson Localization, Tokyo, 1987 (Springer-Verlag, New York, 1988) (in press).

${ }^{12}$ B. L. Al'tshuler and D. E. Khmel'nitskii, Pis'ma Zh. Eksp. Teor. Fiz. 42, 291 (1985) [JETP Lett. 42, 359 (1985)].

${ }^{13}$ A. I. Larkin and D. E. Kheml'nitskii, Zh. Eksp. Teor. Fiz. 91, 1815 (1986) [Sov. Phys._JETP 64, 1075 (1986)] (there are several typographical errors in the formulas in this paper); Phys. Scr. T14, 4 (1986).

${ }^{14}$ S. Kaplan, Surf. Sci. 196, 93 (1988).

${ }^{15} \mathrm{~S}$. Hershfield (unpublished).

${ }^{16}$ A. D. Benoit, C. P. Umbach, R. B. Laibowitz, and R. A. Webb, Phys. Rev. Lett. 58, 2343 (1987); W. J. Skocpol, P. M. Mankiewich, R. E. Howard, L. D. Jackel, D. M. Tennant, and A. D. Stone, ibid. 57, 2347 (1987); 56, 2865 (1986).

${ }^{17}$ H. Baranger, A. D. Stone, and D. P. Divincenzo (unpublished).

${ }^{18}$ C. L. Kane, P. A. Lee, and D. P. Divincenzo (unpublished).

${ }^{19}$ H. Haucke, S. Washburn, A. D. Benoit, C. P. Umbach, and R. A. Webb (unpublished).

${ }^{20}$ S. Washburn, Y. Imry, and R. A. Webb (unpublished). 


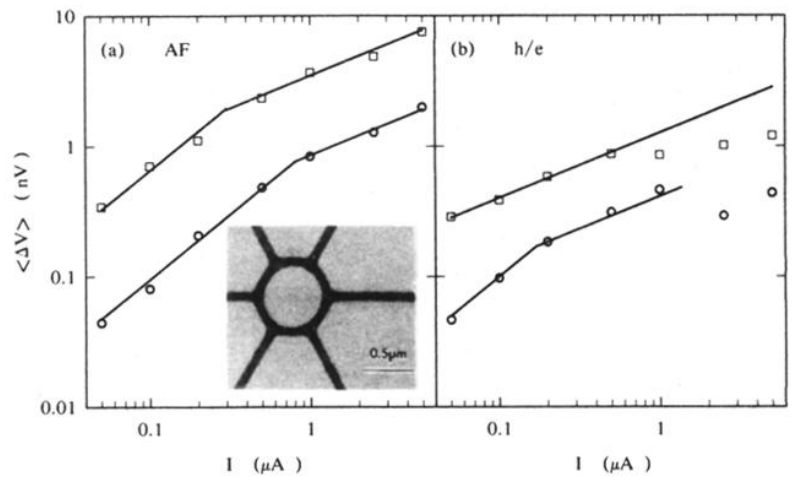

FIG. 3. (a) $\langle\Delta V\rangle$ as a function of the drive current $I$ at the fundamental $(\square)$ and first harmonic $(O)$ for the aperiodic fluctuations. Inset is the measured $0.58 \mu \mathrm{m}$ inside diameter gold loop with six equally spaced probes. (b) $\langle\Delta V\rangle$ as a function of $I$ at the fundamental $(\square)$ and first harmonic $(O)$ for the $h / e$ oscillations. 